\title{
The RMS survey: radio observations of candidate massive YSOs in the southern hemisphere
}

\author{
J. S. Urquhart ${ }^{1}$, A. L. Busfield ${ }^{1}$, M. G. Hoare ${ }^{1}$, S. L. Lumsden ${ }^{1}$, \\ A. J. Clarke ${ }^{1}$, T. J. T. Moore ${ }^{2}$, J. C. Mottram ${ }^{1}$ and R. D. Oudmaijer ${ }^{1}$ \\ ${ }^{1}$ School of Physics and Astrophysics, University of Leeds, Leeds, LS2 9JT, UK \\ email: jsu@ast.leeds.ac.uk \\ ${ }^{2}$ Astrophysics Research Institute, Liverpool John Moores University, Twelve Quays House, \\ Egerton Wharf, Birkenhead, CH41 1LD, UK
}

\begin{abstract}
The Red MSX Source (RMS) survey (Hoare et al. 2005) is a multi-wavelength programme of follow-up observations designed to distinguish between genuine massive young stellar objects (MYSOs) and other embedded or dusty objects, such as ultra compact (UC) HII regions, evolved stars and planetary nebulae (PNe). We have identified nearly 2000 MYSOs candidates by comparing the colours of MSX and 2MASS point sources to those of known MYSOs. There are several other types of embedded or dust enshrouded objects that have similar colours as MYSOs and contaminate our sample. Two sources of contamination are from UCHII regions and $\mathrm{PNe}$, both of which can be identified from the radio emission emitted by their ionised nebulae. In order to identify UCHII regions and PNe that contaminate our sample we have conducted high resolution radio continuum observations at 3.6 and $6 \mathrm{~cm}$ of all southern MYSOs candidates $\left(235^{\circ}<l<350^{\circ}\right)$ using the Australia Telescope Compact Array (ATCA).
\end{abstract}

Keywords. Radio continuum: stars - Stars: formation - Stars: early-type - Stars: pre-main sequence.

\section{Summary and Conclusions}

Observations were made at 3.6 and $6 \mathrm{~cm}$ towards 826 RMS sources located within 802 fields using the ATCA (see Urquhart et al. 2006 for details). These observations were aimed at identifying radio loud contaminants such as UC HII regions and PNe from the relatively radio quiet MYSOs. Of the 826 RMS sources observed we have found 199 to be associated with radio emission $(\sim 25 \%)$. More interestingly we failed to detect any radio emission towards $627 \mathrm{RMS}$ sources, and therefore after eliminating one of the main sources of contamination, we are still left with a large sample of MYSOs candidates. The majority of the 199 RMS sources found to be associated with radio emission are expected to be UCHII regions. The morphologies of these sources, which are consistent with other studies of UCHII regions, relatively flat spectral indices, and their scaleheight would certainly support their identification as UCHII regions. Once combined with the results of our VLA observations we will have an unbiased sample of $\sim 400-500$ UCHII, the vast majority of which were previously known.

\section{References}

Urquhart, J. S., et al. 2006, astro-ph/0605738

Hoare, M. G., et al. 2005, in: R. Cesaroni, M. Felli, E. Churchwell, M. Walmsley, Massive star birth: A crossroads of Astrophysics (Cambridge: Cambridge Univ.), p. 370 